\title{
Pharmacokinetic variability of extended interval tobramycin in burn patients
}

\author{
David Bracco $^{a, *}$, Christine Landry ${ }^{a}$, Marc-Jacques Dubois ${ }^{a}$, Philippe Eggimann ${ }^{b}$ \\ ${ }^{a}$ Montreal Burn Centre, Montreal University Hospital, Campus Hotel Dieu, Montreal, Canada \\ ${ }^{\mathrm{b}}$ ICU and Infectious Disease, Burn Unit, Lausanne University Hospital, Lausanne, Switzerland
}

\section{A R T I C L E I N F O}

Article history:

Received 28 June 2007

Accepted 6 November 2007

\section{Keywords:}

Tobramycin

Pharmacokinetics

Burn

Therapeutic drug monitoring

Nosocomial infection

Aminoglycoside

Critically ill patients

Distribution volume

\begin{abstract}
A B S T R A C T
Background: Aminoglycosides are mandatory in the treatment of severe infections in burns. However, their pharmacokinetics are difficult to predict in critically ill patients. Our objective was to describe the pharmacokinetic parameters of high doses of tobramycin administered at extended intervals in severely burned patients.

Methods: We prospectively enrolled 23 burned patients receiving tobramycin in combination therapy for Pseudomonas species infections in a burn ICU over 2 years in a therapeutic drug monitoring program. Trough and post peak tobramycin levels were measured to adjust drug dosage. Pharmacokinetic parameters were derived from two points first order kinetics. Results: Tobramycin peak concentration was $7.4(3.1-19.6) \mu \mathrm{g} / \mathrm{ml}$ and $C_{\max } / \mathrm{MIC}$ ratio 14.8 (2.8-39.2). Half-life was 6.9 (range 1.8-24.6) $\mathrm{h}$ with a distribution volume of $0.4(0.2-1.0) \mathrm{l} / \mathrm{kg}$. Clearance was 35 (14-121) $\mathrm{ml} / \mathrm{min}$ and was weakly but significantly correlated with creatinine clearance.

Conclusion: Tobramycin had a normal clearance, but an increased volume of distribution and a prolonged half-life in burned patients. However, the pharmacokinetic parameters of tobramycin are highly variable in burned patients. These data support extended interval administration and strongly suggest that aminoglycosides should only be used within a structured pharmacokinetic monitoring program.
\end{abstract}

(C) 2007 Elsevier Ltd and ISBI. All rights reserved.

\section{Introduction}

The pharmacokinetic of many drugs is profoundly altered in burned patients with modifications in both volumes of distribution and drug disposal [1]. The immediate period after burn is characterized by decreased cardiac output and hepatic and renal drug clearances. This phase is followed by an acute physiological response including an intense hypermetabolic phase with the supranormal cardiac output, increased liver and renal perfusion and - usually - increased drug clearances.
These physiologic reactions make dosing antibiotics in burned patients delicate [1]. In addition, previous exposure to antibiotics and increasingly resistant micro-organisms made the choice of the antibiotic and administration regimen crucial. Aminoglycoside treatment remains mandatory for difficult to treat organisms such as Pseudomonas species.

The antibacterial activity of aminoglycosides is strongly dependent on the peak serum level. To achieve clinical success a high peak serum levels to minimum inhibitory concentration (MIC) ratios as well as high area under the curve to MIC (AUC) are necessary [2,3]. Maximal serum concentrations to MIC ratio

\footnotetext{
* Corresponding author at: Department of Anesthesia, McGill University Health Center, Montreal General Hospital, 1650 Cedar Avenue, Room D 10-145-3, Montreal H3G 1A4, Canada. Tel.: +1 514934 1934x43030; fax: +1 5149348249.

E-mail address: David.Bracco@McGill.Ca (D. Bracco). 
( $C_{\text {max }} /$ MIC ratio) above 10 are recommended. Aminoglycosides are also characterized by a prolonged post-antibiotic effect, defined as a delayed regrowth of bacteria following exposure to an antibiotic despite serum concentrations decreasing below the MIC. The duration of this post-antibiotic effect against gram-negative bacteria ranges from 1 to $4 \mathrm{~h}$. The nephrotoxicity of aminoglycosides is mostly related to high trough concentrations and interval between doses. This has brought the concept of giving aminoglycosides at high doses at extended intervals. Numerous clinical studies validated this approach in non-burn patients and several meta-analyses confirm its value. Despite good level of evidence, reported clinical experience in critically ill and burn patients is disappointing [4-8].

The aims of this observational study were (1) to describe the pharmacokinetic parameters of extended interval dosing (EID) tobramycin in burned patients enrolled in a therapeutic drug monitoring (TDM) program and (2) to explore the determinants of pharmacokinetic parameters in severely burned patients.

\section{Material and methods}

The Montreal Burn Centre is a 10 bed burn ICU serving an area of 8 million inhabitants and admitting approximately 150 major burns each year (mean burn surface area: $22 \%$ ). The ethical committee of the hospital approved the study. Informed consent from the patient or relative was waived since this study was considered as clinical performance improvement (Protocol \#HD 04.087).

Between 1st July 2004 and 30th June 2006, patients in whom a combination treatment including an aminoglycoside was required for nosocomial infections were prospectively enrolled. Extended interval dosage was recommended. If the dose of tobramycin was above $200 \mathrm{mg}$, it was infused over $1 \mathrm{~h}$ and in $30 \mathrm{~min}$ otherwise. Antibiotic drug concentrations were performed routinely after the 3rd dose and dosages adapted to optimize peak and trough levels. The distribution half-life $\left(T_{1 / 2} \alpha\right)$ of tobramycin is between 0.36 and $0.5 \mathrm{~h}$. Peak levels drawn $30 \mathrm{~min}$ after infusion termination reflects this distribution phase and thus a one compartment model may be inadequate. To avoid this problem and focus on the true elimination phase, peak levels were drawn $2 \mathrm{~h}$ after infusion end. Trough levels were drawn just before the next dose. Serum tobramycin level were measured by Fluorescence Polarization Immunoassay [9] (Abbott TDX, Abbott Laboratories. Abbott Park, Illinois, USA).

The admission and actual body weight, plasma levels of creatinine and albumin on the day of TDM assessment were also recorded allowing estimation of the creatinine clearance using the Cockroft-Gault equation.

Drug levels were analyzed using a custom made plug-in (4D view language; version 2003.5, ACI) part of our clinical information database. The pharmacokinetic model is based on a first order one compartment kinetic. Elimination rate constant, plasma half-life, clearance, maximal $\left(C_{\max }\right)$ and minimal $\left(C_{\min }\right)$ concentrations, AUC, $C_{\max } / \mathrm{MIC}$ ratio and volume of distribution were calculated using the equations described in the appendix. Based on these parameters the optimal dosage and interval were calculated to achieve a peak concentration of $8 \mathrm{mg} / \mathrm{l}$ and levels below the MIC at $4 \mathrm{~h}$ (postantibiotic effect). With an MIC of $0.5 \mu \mathrm{g} / \mathrm{ml}$ for tobramycinsensitive micro-organisms, this would provide a $C_{\max }$ to MIC ratio of above 16 .

The plasma tobramycin concentration over time of a standard $5 \mathrm{mg} / \mathrm{kg}$ once daily dose [10] administered to all burn patients was calculated in each patient.

Data were analyzed using the SAS statistical platform. Due to the reduced sample size, non-parametric tests were applied and the population data are presented as median and range. Maximal concentration $\left(C_{\max }\right)$, clearance, and volumes of distribution of tobramycin were compared with values

Table 1 - Demographic data

Proportions or median (range)

Gender

Age (years)

Weight on the TDM day $(\mathrm{kg})$

Burn surface area (\%)

Surgical burned area (\%)

Cause of injury

Associated inhalation injury

Delay from burns (days)

Mechanical ventilation

Tobramycin dose (mg)

Administration interval $(\mathrm{h})$

Plasma creatinine level $(\mu \mathrm{mol} / \mathrm{l})$

Plasma albumin level $(\mathrm{g} / \mathrm{l})$

Estimated creatinine clearance $(\mathrm{ml} / \mathrm{min})$

Microorganisms requiring combined therapy

Infected site: $n(\%)$

\section{$10 \mathrm{~F} / 13 \mathrm{M}$}

61 (19-77)

$63.4(41.6-101.9)$

$53(10-70)$

$48(10-70)$

Thermal burn: $91 \%$

Electrical burn: $9 \%$

$10 / 23(43 \%)$

$33(8-87)$

$14 / 23(61 \%)$

$260(80-350)$

$24(18-36)$

$57(29-116)$

$27(14-38)$

96.1 (38.8-261.9)

Pseudomonas aeruginosa $96 \%$

Stenotrophomonas maltophilia $4 \%$

Skin: 19 (83\%)

Lung: 3 (13\%)

Bloodstream infection: $1(4 \%)^{\mathrm{a}}$

a 1 case, presumably from a burn wound infection. 


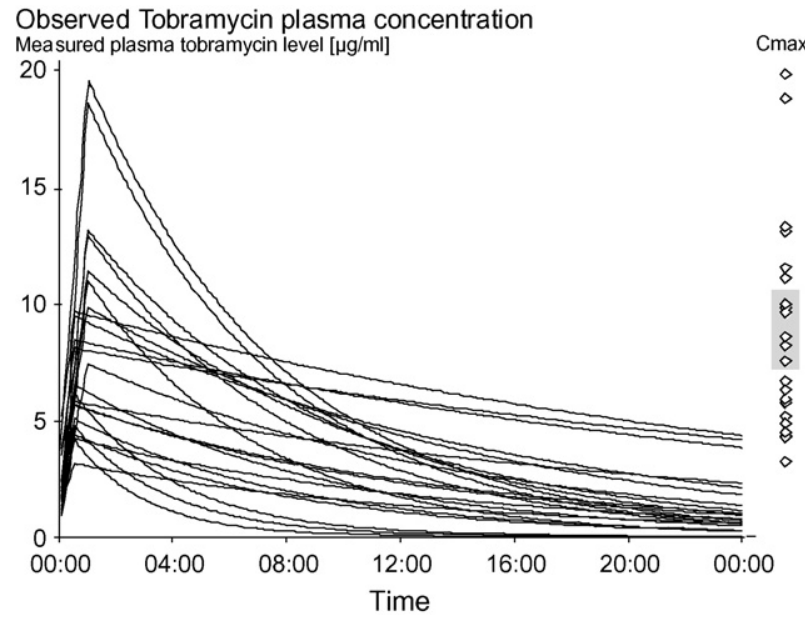

Fig. 1 - Measured tobramycin levels.

reported for a non-burn population [11] using the Rank Sum test. Observed and simulated serum tobramycin concentration were plotted against time by running each patient's observed and simulated concentrations with a 1 min step. Pharmacokinetic parameters were explored against population descriptors by univariate analysis. Due to reduced sample size no multivariate analysis was performed. A $P$ value lower than 0.05 was considered significant.

\section{Results}

During the 2 years period a total of 287 patients were seen in the burn unit. A total of 23 patients receiving tobramycin were included in the TDM program over 2 years. The characteristics of these patients are presented in Table 1 . No patient had hepatic failure or required extra-corporeal renal support.

\section{Table 2 - Pharmacokinetic data}

\begin{tabular}{|c|c|}
\hline & Median (range) \\
\hline Measured through tobramycin level (mg/l) & $1.0(0.2-4.4)$ \\
\hline Measured $2 \mathrm{~h}$ post administration level (mg/l) & $6.3(2.1-14.6)$ \\
\hline Elimination half-life $(\mathrm{h})$ & $6.9(1.8-24.6)$ \\
\hline Tobramycin clearance (ml/min) & $35.3(14.3-120.9)$ \\
\hline (\% of creatinine clearance) & $38(10-110)$ \\
\hline Calculated $C_{\max }(\mathrm{mg} / \mathrm{l})$ & $7.4(3.1-19.6)$ \\
\hline $\mathrm{C}_{\max } / \mathrm{CMI}$ ratio & $14.8(2.8-39.2)$ \\
\hline Calculated time to MIC (h) & $26.1(5.9-99.3)$ \\
\hline \multicolumn{2}{|l|}{ Post antibiotic effect } \\
\hline Through level above MIC & $15 / 23$ \\
\hline Through level below MIC & $7 / 23$ \\
\hline Time below MIC (in $7 / 23$ patients) (h) & $4.8(0.7-10.1)$ \\
\hline $\mathrm{C}_{\max }$ to MIC ratio & $14.8(2.8-39.2)$ \\
\hline Distribution volume (l) & $26.5(11.7-54.3)$ \\
\hline Distribution volume $(1 / \mathrm{kg})$ & $0.4(0.2-1.0)$ \\
\hline AUC $\left(\mathrm{mg} / l^{*} \mathrm{~h}\right)$ & $86.6(11.8-161.1)$ \\
\hline Optimal dose (mg) & $260(113-525)$ \\
\hline Optimal dose (mg/kg) & $3.8(2.3-9.2)$ \\
\hline Optimal dosing interval (h) & $36(9.5-120)$ \\
\hline
\end{tabular}

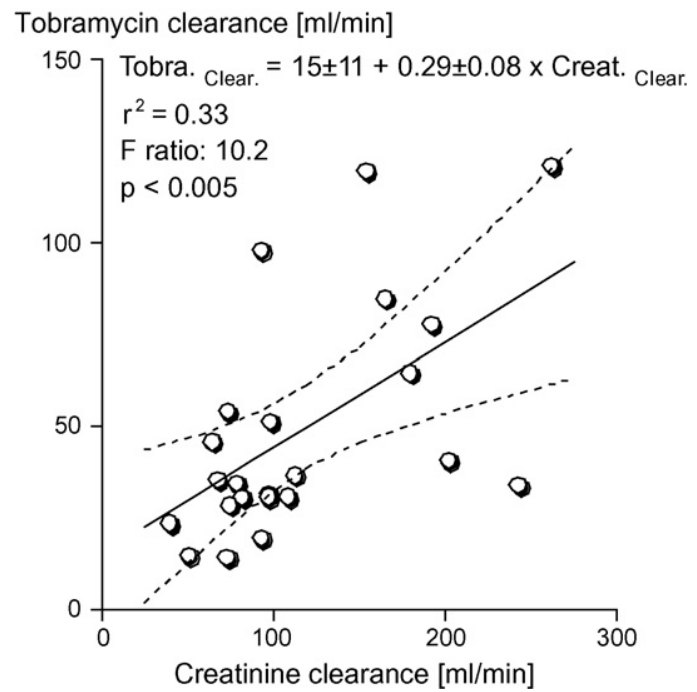

Fig. 2 - Correlation between creatinine and tobramycin clearances.

Tobramycin daily dose ranged from 80 to $350 \mathrm{mg}$ with intervals from 16 to $36 \mathrm{~h}$. Trough and peak concentrations of tobramycin are shown in Table 2 . There was a great variation of peak plasma concentration and volumes of distribution (Fig. 1). Only 35\% (8/23) had peak serum levels that were in the optimal range (grey box), peak levels were below in 39\% (9/23) and were above the optimal range in $26 \%$ (6/23 of the patients). In $70 \%(16 / 23)$ of patients, the tobramycin plasma level remained above $0.5 \mathrm{mg} / \mathrm{l}$ at all times. Time below CMI was between $40 \mathrm{~min}$ and $10 \mathrm{~h}$ in the remaining seven patients. The median tobramycin clearance was found to be $38 \%$ of the median creatinine clearance (Fig. 2). There was a weak but significant correlation between tobramycin and creatinine clearances. No patient developed renal failure, and the plasma creatinine levels were not modified by tobramycin administration. Decreasing plasma levels of albumin correlated with a

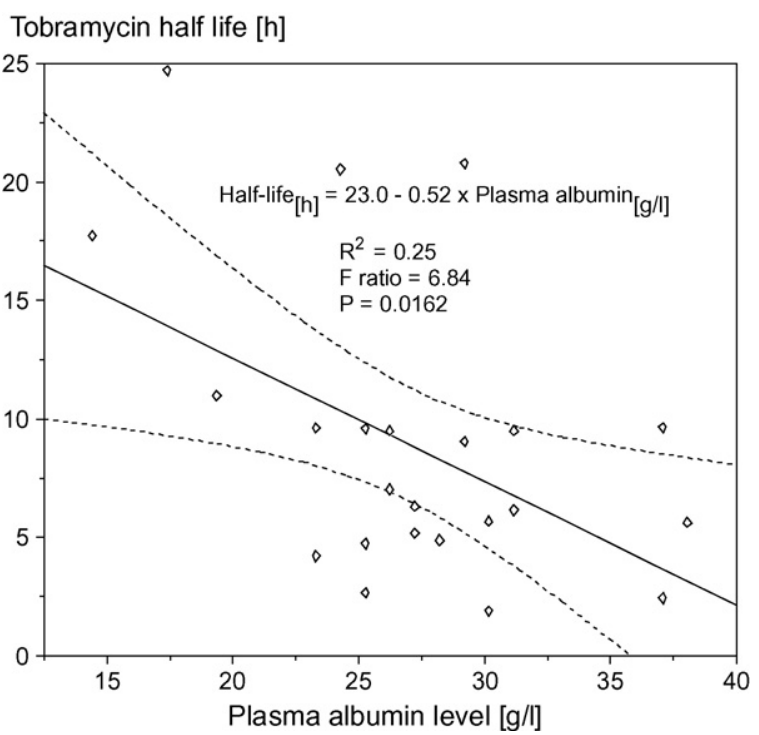

Fig. 3 - Correlation between plasma albumin level and tobramycin half-life. 


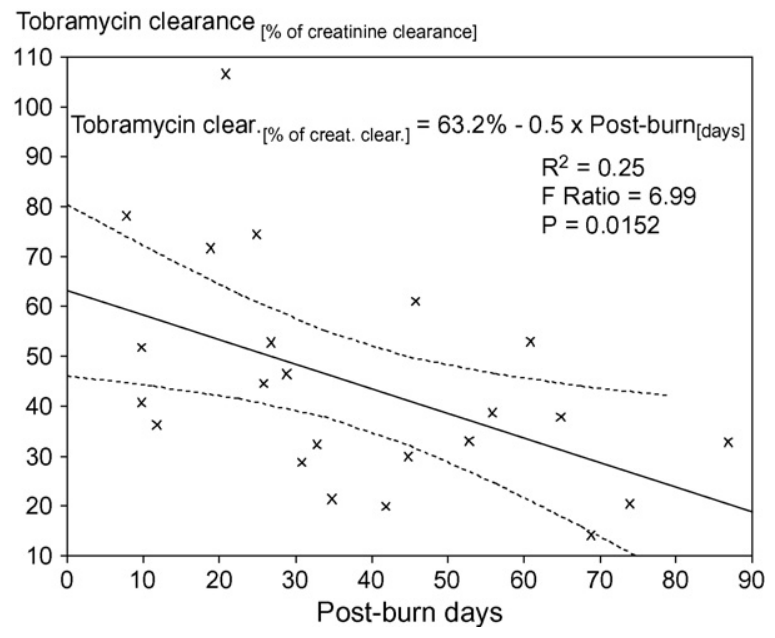

Fig. 4 - Tobramycin clearance and days post-burn.

prolonged tobramycin half-life (Fig. 3). Tobramycin clearanceexpressed as a proportion of creatinine clearance - showed an inverse relationship with the post-burn delay (Fig. 4).

There was no correlation between the volume of distribution and the day after burn, fluid accumulation, burn surface area, creatinine or albumin plasma levels.

To reach a target $C_{\max }$ of $8 \mu \mathrm{g} / \mathrm{ml}$ and $4 \mathrm{~h}$ below the MIC, the median optimal dose would be $260 \mathrm{mg}$ (range $113-525 \mathrm{mg}$ ) or $3.8 \mathrm{mg} / \mathrm{kg}$ (range $2.3-9.2 \mathrm{mg} / \mathrm{kg}$ ). A median administration interval of $36 \mathrm{~h}$ (range 9.5-120 h) would be required.

The extrapolated time course of plasma tobramycin concentrations following a "flat rate" of $5 \mathrm{mg} / \mathrm{kg}$ of tobramycin every $24 \mathrm{~h}$ showed a wide variation in peak and through levels (Fig. 5). Only 48\% (11/23) peak plasma levels were in the suggested therapeutic target, 48\% (11/23) peak levels being above the optimal target and one was insufficient.

\section{Discussion}

Extended interval aminoglycosides regimens are progressively accepted as the preferred administration schedule in

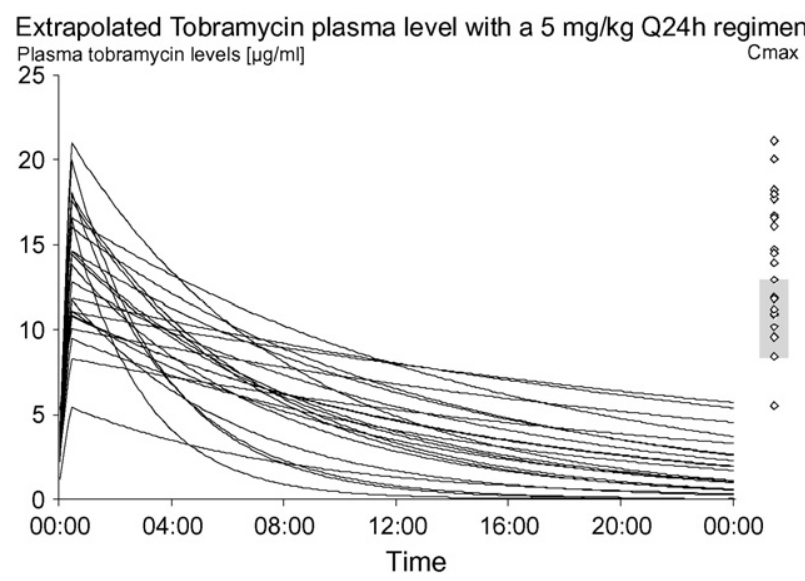

Fig. 5 - Extrapolated plasma tobramycin levels following a standard $5 \mathrm{mg} / \mathrm{kg}$ Q24 $\mathrm{h}$ regimen in a burn population. critically ill patients. However, there is little information on the pharmacokinetic of extended interval administration of aminoglycosides in burn patients [8]. The main findings of the present study were an important increase in distribution volume and in elimination half-life with a near normal tobramycin clearance.

Tobramycin binding to protein is below 30\% [12]. Low plasma albumin levels may interfere with pharmacodynamic properties of aminoglycosides, although this has never been demonstrated for tobramycin. We observed a prolongation of the half-life and an increase in distribution volume, suggesting a almost unchanged in clearance of tobramycin. Tobramycin clearance was lower than previously reported in burned patients receiving 5-7 mg/kg of gentamycin or tobramycin [8].

Aminoglycosides are filtered and partly reabsorbed in proximal tubular cells [13]. This reabsorption mechanism induces an accumulation of tobramycin in cortical renal cells, contributing to its nephrotoxic effect. Tobramycin reabsorption is, in part, coupled with megalin which is a non specific proximal tubular reabsorption molecule [14]. One may speculate that due to increased glomerular endothelial permeability more tobramycin reaches the proximal tubule. The megalin complex could be saturated and cannot reabsorb the increased tubular load of aminoglycosides, increasing its elimination.

Peris-Marti et al. [15] measured tobramycin pharmacokinetics in 51 critically ill patients and calculated the pharmacokinetic parameters. They found an adequate $C_{\max }$ and $C_{\max }$ to MIC ratio but found an increased time below MIC. This time below MIC exceeded the duration of the post-antibiotic effect, exposing to a risk of bacterial proliferation. Buijk et al. [16] pooled the TDM data from ICU patients receiving gentamicin $(n=66)$ or tobramycin $(n=23)$. The mean distribution volume was $0.31 \mathrm{l} / \mathrm{kg}$, with a clearance of $83 \mathrm{ml} / \mathrm{min}$ and an half-life of $4.0 \mathrm{~h}$. They found a wide variability of aminoglycoside pharmacokinetic in critically ill patients. Barletta et al. investigated TDM of tobramycin in 19 critically ill patients [5]. The mean observed distribution volume was $0.31 / \mathrm{kg}$ and half-life $2.9 \mathrm{~h}$. Dorman et al. [7] measured tobramycin and gentamicin levels in 53 ICU patients. They also found an increase in distribution volume suggesting that larger loading doses may be required.

Recently Conil et al. [4] measured the pharmacokinetic parameter of $20 \mathrm{mg} / \mathrm{kg}$ amikacin in 38 adult burn patients. They showed that these burns requires higher than normal doses, and also exhibit a large interindividual variability. The present data confirm Conil's investigation showing that burn patients have an increased distribution volume for aminoglycosides, and that the aminoglycoside clearance is proportional to the creatinine clearance. Conil [4] showed an inverse relationship between the burn surface area and the aminoglycoside clearance mainly due to very high clearance in less severe burns. As no patients with $\mathrm{BSA}<10 \%$ were included in our population we were unable to show a relationship between BSA and tobramycin clearance.

Hoey et al. [8] investigated single daily doses of aminoglycosides (gentamicin or tobramycin) in 52 burned patient. They found a wide variability of $C_{\max }$ and time to MIC, and concluded that burned patients were not good candidates 
for single daily dose aminoglycosides. Our data confirmed the high variability of tobramycin pharmacokinetic parameters but showed that dosage and interval of administration may be optimized with a strict TDM program.

The major limitations of the study were the small number of patients, the wide variation of the administration protocols and the clinical conditions of these patients. The use of estimated value of creatinine clearance may limit the value of comparison of tobramycin and creatinine clearance. However, antibiotic doses were not adapted to the creatinine clearance. The kinetic calculations were based only on two points, and this may decrease the precision of the model. Complete pharmacokinetic exploration of aminoglycosides includes three compartments: (1) A rapid initial redistribution compartment with a half-life of $0.36-0.5 \mathrm{~h}$. (2) A true elimination phase explored in the present study and (3) A so-called "deep" compartment reflecting tissue fixation with a half-life of $200 \mathrm{~h}$ [17]. We hypothesized that the contribution of this "deep" compartment was negligeable in the present study. Patients were enrolled from 8 to 87 days after major burn. Although they had a variable post-burn time, they all shared a severe sepsis with multi-resistant organisms.

\section{Conclusions}

The pharmacokinetic of tobramycin is difficult to predict in critically burned patients. The increased volume of distribution leads to suboptimal $C_{\max }$ and $C_{\max } / \mathrm{MIC}$ ratio, whereas prolonged elimination half-life exposes the patient to high through concentration and increased risk of oto- and nephrotoxicity. Our data shows that close therapeutic drug monitoring is strongly suggested for tobramycin use in severely burned patients to optimize the $\mathrm{C}_{\max } / \mathrm{MIC}$ ratio and the duration at low concentration. In this context, these drugs remain an excellent choice in difficult to treat burn infections.

\section{Conflict of interest statement}

None.

\section{Appendix A. Pharmacokinetic equations used}

\author{
Elimination slope



Elimination half-life

$$
\begin{aligned}
& \mathrm{T}_{1 / 2}=\frac{0.693}{\lambda} \\
& \text { Maximal concentration } \\
& \text { Conc }_{\max }=\frac{\text { conc }_{\text {measured post-dose }}}{\mathrm{e}^{\lambda \times \Delta \mathrm{Time}_{(\text {endinfusion } \rightarrow \text { post }} \text { sampling) }}}
\end{aligned}
$$

Concentration function

Conc $_{\text {time } i}=$ conc $_{\max } \times \mathrm{e}^{\lambda \times \Delta \text { Time }_{(\text {Endinfusion } \rightarrow \text { Timei })}}$

Residual concentration

Conc $_{\text {resid }}=$ conc $_{\max } \times \mathrm{e}^{\lambda \times \Delta \text { Time }_{(\text {end infusion } \rightarrow \text { start next infusion })}}$

Volume of distribution

Volume of distribution

$$
=\frac{\text { perfusion speed }_{[\mathrm{mg} / \mathrm{h}]} \times\left(1-\mathrm{e}^{\lambda \times \text { perfusion duration }}\right)}{\lambda \times\left(C_{\max }-C_{\min } \times \mathrm{e}^{(\lambda \times \text { perfusion duration })}\right)}
$$

Tobramycin clearance

Clearance $=\frac{0.693 \times \text { distribution volume }}{\text { elimination half-life }}$

REFERENCES

[1] Weinbren MJ. Pharmacokinetics of antibiotics in burn patients. J Antimicrob Chemother 1999;44:319-27.

[2] Burton ME, Ash CL, Hill Jr DP, Handy T, Shepherd MD, Vasko MR. A controlled trial of the cost benefit of computerized bayesian aminoglycoside administration. Clin Pharmacol Ther 1991;49:685-94.

[3] Vinks AA, Evers N, Mathot R, Michel B. Impact of goaloriented model-based tdm of aminoglycosides on clinical outcome: a cost-effectiveness analysis. Ther Drug Monit 1997;19:547 [Abstract].

[4] Conil JM, Georges B, Breden A, Segonds C, Lavit M, Seguin T, et al. Increased amikacin dosage requirements in burn patients receiving a once-daily regimen. Int J Antimicrob Agents 2006;28:226-30.

[5] Barletta JF, Johnson SB, Nix DE, Nix LC, Erstad BL. Population pharmacokinetics of aminoglycosides in critically ill trauma patients on once-daily regimens. J Trauma 2000;49:869-72.

[6] Chicano-Pia PV, Cercos-Lleti AC, Roma-Sanchez E. Pharmacokinetic model for tobramycin in acinetobacter meningitis. Ann Pharmacother 2002;36:83-6.

[7] Dorman T, Swoboda S, Zarfeshenfard F, Trentler B, Lipsett PA. Impact of altered aminoglycoside volume of distribution on the adequacy of a three milligram per kilogram loading dose. Critical care research group. Surgery 1998;124:73-8.

[8] Hoey LL, Tschida SJ, Rotschafer JC, Guay DR, Vance-Bryan $\mathrm{K}$. Wide variation in single, daily-dose aminoglycoside pharmacokinetics in patients with burn injuries. J Burn Care Rehabil 1997;18:116-24.

[9] Ngui-Yen JH, Doyle PW, Smith JA. Comparative analysis of two rapid, automated methods for determining aminoglycoside levels. J Clin Microbiol 1984;20: 962-5.

[10] Gilbert DN, Moellering RC, Ellopoulos GM, Sande MA. The Sanford guide to antimicrobial therapy 2007, 37th ed., P.O. Box 276, 11771 Lee Highway, Sperryville, VA 22740-0276, USA: Antimicrobial Therapy Inc.; 2007.

[11] Turnidge J. Pharmacodynamics and dosing of aminoglycosides. Infect Dis Clin North Am 2003;17:503-28.

[12] Bailey DN, Briggs JR. Gentamicin and tobramycin binding to human serum in vitro. J Anal Toxicol 2004;28:187-9. 
[13] Nagai J, Takano M. Molecular aspects of renal handling of aminoglycosides and strategies for preventing the nephrotoxicity. Drug Metab Pharmacokinet 2004;19: 159-70.

[14] Birn H, Fyfe JC, Jacobsen C, Mounier F, Verroust PJ, Orskov $\mathrm{H}$, et al. Cubilin is an albumin binding protein important for renal tubular albumin reabsorption. J Clin Invest 2000;105:1353-61.

[15] Peris-Marti JF, Borras-Blasco J, Rosique-Robles JD, GonzalezDelgado M. Evaluation of once daily tobramycin dosing in critically ill patients through Bayesian simulation. J Clin Pharm Ther 2004;29:65-70.

[16] Buijk SE, Mouton JW, Gyssens IC, Verbrugh HA, Bruining HA. Experience with a once-daily dosing program of aminoglycosides in critically ill patients. Intensive Care Med 2002;28:936-42.

[17] Tod MM, Padoin C, Petitjean O. Individualising aminoglycoside dosage regimens after therapeutic drug monitoring: simple or complex pharmacokinetic methods? Clin Pharmacokinet 2001;40:803-14. 